\title{
ON CERTAIN SEQUENCE SPACES ॥
}

\author{
HÜSNÜ KIZMAZ \\ Karadeniz Technical University \\ Department of Mathematics \\ 61080 Trabzon, Turkey \\ (Received February 2, 1994 and in revised form July 1, 1994)
}

ABSTRACT in this paper we define the space $c_{0}(\Lambda)=\left\{x=\left(x_{k}\right) / x_{k}-k_{k-1} \rightarrow 0(k \rightarrow \infty), x_{0}=0, x_{k} \in \mathbb{C}\right\}$ and compute its duals. (Continuous dual, $\beta$-dual and $\mathrm{N}$-dual) The aim of this paper is to give same results about matrix mapping of $c_{o}(\Lambda)$ into other sequence spaces including the convergent sequences, null sequences and bounded sequences

KEY WORDS AND PHRASES: Sequence spaces, matrix maps, $\Delta$-norm, $\beta$-dual, Null-dual 1991 AMS SUBJECT CLASSIFICATION CODES: $40 \mathrm{C05}$

\section{Introduction}

Let $I_{\infty}, c$ and $c_{0}$ be the linear spaces of complex bounded, convergent and null sequences $x=\left(x_{k}\right)$ respectively, normed by

$$
\|x\|_{\infty}=\sup _{k}\left|x_{k}\right|
$$

where $k \in \mathbb{N}=\{1,2, \ldots\}$ the positive integers. On the other hand we defined $I_{\infty}(\Delta)=\left\{x=\left(x_{k}\right) / \Delta x \in I_{\infty}\right\}, c(\Delta)=\left\{x=\left(x_{k}\right) / \Delta x \in c\right\}$ and $c_{0}(\Delta)=\left\{x=\left(x_{k}\right) / \Delta x \in c_{0}\right\}$ where $\Delta x=\left(x_{k}-x_{k-1}\right), x_{0}=0$ [2]

(Throughout this paper it is assumed that $x_{0}=0$ )

$c_{0}(\Delta), c(\Delta)$ and $I_{\infty}(\Delta)$ are Banach Spaces with the norm

$$
\|x\|_{\Delta}=\sup _{k}\left|x_{k}-x_{k-1}\right|
$$

$c_{0}, c, I_{\infty}$ and $M_{0}=I_{\infty} \cap c_{0}(\Delta)$ are Banach with the norm $\|.\|_{\infty}$ but they aren't Banach with the norm \|\|$_{\Delta}$.

If we say $s x=\left(\sum_{k=1}^{n} x_{k}\right)$ then we have $m_{s}=\left\{x=\left(x_{k}\right) / s x \in I_{\infty}\right\}, c_{s}=\left\{x=\left(x_{k}\right) / s x \in c\right\}$ and $\left(c_{0}\right) s=\left\{x=\left(x_{k}\right) / s x \in c_{0}\right\}$ [4]. $I_{\infty}, c$ and $c_{0}$ are isometrically isomorphic to $m_{s^{\prime}} c_{s}$ and $\left(c_{0}\right)_{s}$, respectively with their natural norms. For instance $f: I_{\infty} \rightarrow m_{s}, f(x)=\Delta x$ and $f^{-1}: m_{s} \rightarrow I_{\infty}$

$f^{-1}(x)=s x$ are isometric isomorphisms. Similary $I_{\infty}(\Delta), c(\Delta)$ and $c_{0}(\Delta)$ are isometrically isomorphic to $I_{\infty}$. $c$ and $c_{0}$ respectively. Obviously

$$
f:\left(c_{0}(\Delta),\|.\|_{\Delta}\right) \rightarrow\left(c_{0},\|\cdot\|_{\infty}\right), f(x)=\Delta x
$$

and

$$
f-1:\left(c_{0},\|\cdot\|_{\infty}\right) \rightarrow\left(c_{0}(\Delta),\|\cdot\|_{\Delta}\right), \quad f(x)=s x
$$

are isometric isomorphisms.

We have investigated matrix maps and related questions connected with $I_{\infty}(\Delta)$ and $c(\Delta)$ in [2]. We know that ${ }_{k} c_{0}$ and $c$ have Schauder basis but $I_{\infty}$ has no basis with the norm $\|.\|_{\infty}$. Write $\theta_{k}$ $=\left(0,0, \ldots, 0, \frac{\hat{1}}{,}, 0, \ldots\right)$. Then $\left(\theta_{k}\right)$ is a basis for $c_{0}$ and $\left(\theta_{k-1}\right)\left(\theta_{0}=(1,1,1, \ldots)\right)$ is a basis for $c$, with $\|.\|_{\infty}$ and $\|.\|_{\Delta}$. On the other hand $\left.\left(E_{k}\right)=(0,0, \ldots, 0,1,1,1, \ldots)\right)$ is a basis for $M_{0}$ and $c_{0}(\Delta)$ with the norm \|\|$_{\Delta}$. So $c_{0}(\Delta)$ is a separable Banach Space.

We know that the contınuous dusi of $c_{0}$ and $c$ is $I_{1}=\left\{x=\left(x_{k}\right) / \sum_{k=1}^{\infty}\left|x_{k}\right|<\infty, x_{k} \in C\right\}[3]$ (Page 110) (C

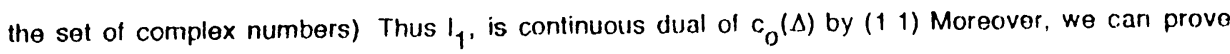
that 


$$
\bar{C}=\bar{M}_{0}=c_{0}(\Delta)
$$

with the norm \|\|$_{i}$, where the bar denoles closure. For this, let $x \in c_{0}(\Delta)$ and $\varepsilon>0$ be any number Then there exists one and only one $y=\left(y_{k}\right) \in c_{0}$ such that $x_{k}=\sum_{i=1}^{k} y_{i}(1.1)$ and a corresponding index $M=M(c) \in \mathbb{N}$ such that $l_{k} \mid<\varepsilon / 2$ for all $k \geq M$. Now we lake

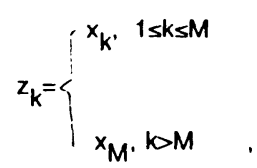

thus $z=\left(z_{k}\right) \in c C c_{0}(\Delta)$ belongs to the open ball $B(x, \varepsilon)$ which is in $\left(c_{0}(\Delta),\|.\|_{\Delta}\right)$

\section{2. $\beta$-dual, N-dual and Matrix Maps}

If $X$ is a sequence space, we define

$x^{\beta}=\left\{a=\left(a_{k}\right) / \sum_{k=1}^{\infty} a_{k} x_{k}\right.$ is Convergent for each $\left.x \in X\right\}$

$x^{N}=\left\{a=\left(a_{k}\right) / \lim _{k} a_{k} x_{k}=0\right.$, for each $\left.x \in X\right\} . X^{\beta}$ is called the $\beta$-(or generalized Köthe-Toeplitz) dual [1] and we will say that $X^{N}$ is $N$-(or null) dual space of $X$. We have that if $X \subset Y$. then $Y \beta_{C} \subset X^{\beta}$. The $N$-dual has similar properties with the $\beta$-dual. For instance if $X \subset Y$ than $Y^{N} \subset X^{N}$ and $X \beta \subset X N$.

$$
\text { Obviously } c_{0}^{N}=I_{\infty}, I_{\infty}^{N}=M_{0}^{N}=c^{N}=c_{0} \text {, }
$$

$c^{N}(\Delta)=I_{\infty}^{N}(\Delta)=\left\{a=\left(a_{k}\right) /\left(k a_{k}\right) \in c_{0}\right\}$. Let $(X, Y)$ denote the set of all infinite matrices $A=\left(a_{n k}\right)$ which map $X$ into $Y$.

LEMMA 1. Let $\left(a_{k}\right) \in l_{1}$ and if $\lim _{k}\left|a_{k} x_{k}\right|=L$ exists for an $x \in c_{0}(\Delta)$, than $L=0$.

Proof. It is trivial if $x=\left(x_{k}\right)$ is bounded. Suppose that $x \in c_{0}(\Delta)$ is unbounded and $\lim _{k}\left|a_{k} x_{k}\right|=L>0$.

Then $x$ can't have a bounded subbequence. II $\left(x_{k_{n}}\right)$ is bounded then $\lim _{n}\left|a_{k_{n}} x_{k_{n}}\right|=0$ implies $L=0$. So we can take $x_{k}=$ for all $n \in I N$.

Now let $\varepsilon=\frac{L}{2}>0$, than there exists an $M_{1}=M_{1}(\varepsilon) \in \mathbb{N}$ such that $\frac{L}{2}<\left|a_{k} x_{k}\right|<\frac{3 L}{2}$ for all $k \geq M_{1}$. Thus we get $\left|a_{k}\right|>\frac{L}{2} \frac{1}{\left|x_{k}\right|}$ for all $k \geq M_{1}$ and

$$
\sum_{k=1}^{\infty} \frac{1}{\left|x_{k}\right|}<\infty
$$

We have that $\frac{x_{k}}{k} \rightarrow 0(k \rightarrow \infty)$ [2]. Let $c=1$, then we have $\frac{\left|x_{k}\right|}{k}<1$ and $\frac{1}{\left|x_{k}\right|}>\frac{1}{k}$ for all $k \geq M_{2}(1) \in \mathbb{N}$. If we take $\max \left\{M_{1}, M_{2}\right\}=M$ then $\sum_{k=1}^{\infty} \frac{1}{\left|x_{k}\right|} \geq \sum_{k=M}^{\infty} \frac{1}{\left|x_{k}\right|}=\infty$. This contradicts with (2.1). So $L$ must be zero.

LEMMA 2. $c_{0}^{N}(\Delta)=\left\{a=\left(a_{k}\right) /\left(k a_{k}\right) \in I_{\infty}\right\}=E$.

Proof. Suppose that $a=\left(a_{k}\right) \in E$. Since $\lim _{k} \frac{x_{k}}{k}=0$ for all $x=\left(x_{k}\right) \in c_{0}(\Delta)$ [2], then we get $\lim _{k} a_{k} x_{k}=\lim _{k} k a_{k} \frac{x_{k}}{k}=0$. This implies that $a \in c_{0}^{N}(\Delta)$.

Now let $a \in c_{0}^{N}(\Delta)$. Then $\lim _{k} a_{k} x_{k}=0$, for all $x \in c_{0}(\Delta)$, then there exists one and only one $y=\left(y_{k}\right) \in c_{0}$, 
such that $x_{n}=\sum_{k=1}^{n} y_{k}\left(\begin{array}{ll}1 & 1\end{array}\right)$

$\lim _{n} a_{n} x_{n}=\lim _{n} \sum_{k=1}^{n} a_{n} y_{k}=0$ for all $y=\left(y_{k}\right) \in c_{0}$ If we take

we get $\lim _{n} \sum_{k=1}^{\infty} a_{n k} y_{k}=0$, for all $x \in c_{0}$. Then $A=\left(a_{n k}\right) \in\left(c_{0}, c_{0}\right)$ and we have

$$
a_{n k}= \begin{cases}a_{n}, & 1 \leq k \leq n \\ 0, & k>n\end{cases}
$$

$\operatorname{Sup}_{n} \sum_{k=1}^{\infty}\left|a_{n k}\right|=\sup _{n} \sum_{k=1}^{n}\left|a_{n}\right|=\operatorname{Sup}_{n} n\left|a_{n}\right|<x \quad$ [4] This completes the proot

For the next results we introduce the sequence $\left(R_{k}\right)$ [resp matrix $R$ ] given by $R_{k}=\sum_{i=k}^{\infty} a_{1}$ [resp matrix $\left.R=\left(R_{n k}\right)=\left(\sum_{i=k}^{\infty} a_{n i}\right)\right]$

LEMMA 3. $c_{0}^{\beta}(\Delta)=\left\{a=\left(a_{k}\right) \in I_{1} /\left(R_{k}\right) \in I_{1} \cap c_{0}^{N}(\Delta)\right\}=D$

Proof. Suppose that $a \in D$ If $x \in c_{0}(\Delta)$ then we use Abel's summation formula to get

$$
\begin{aligned}
& \sum_{k=1}^{n} a_{k} x_{k}=\sum_{k=1}^{n}\left(\sum_{i=1}^{k} a_{1}\right)\left(x_{k}-x_{k+1}\right)+\left(\sum_{k=1}^{n} a_{k}\right) x_{n+1} \\
& =\sum_{k=1}^{n}\left(R_{1}-R_{k+1}\right)\left(x_{k}-x_{k+1}\right)+\left(R_{1}-R_{n+1}\right) x_{n+1} \\
& =\sum_{k=1}^{n+1} R_{k}\left(x_{k}-x_{k-1}\right)-R_{n+1} x_{n+1}
\end{aligned}
$$

This implies that $\sum_{k=1}^{\infty} a_{k} x_{k}$ is convergent, then $a \in c_{0}^{\beta}(\Delta)$.

If $a \in c_{0}^{\beta}(\Delta)$ then $\sum_{k=1}^{\infty} a_{k} x_{k}$ is convergent for all $x \in c_{0}(\Delta)$ Obviously $a \in l_{1}$ if $x \in c_{0}(\Delta)$, then there

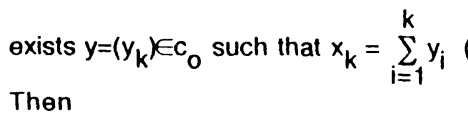

$$
\begin{gathered}
\sum_{k=1}^{n} R_{k} y_{k}=\sum_{k=1}^{n}\left(\sum_{i=1}^{k} y_{i}\right) a_{k}+R_{n+1} \sum_{k=1}^{n} y_{k} \text { with Abel summation formula Thus we have } \\
\qquad \sum_{k=1}^{n} a_{k} x_{k}=\sum_{k=1}^{n}\left(R_{k}-R_{n+1}\right) y_{k}=\sum_{k=1}^{n}\left(\sum_{i=k}^{n} a_{i}\right) y_{k}
\end{gathered}
$$

If we take

$$
a_{n k}=\left\{\begin{array}{cc}
\sum_{i=k}^{n} a_{i}, & 1 \leq k \leq n \\
0, & k>n
\end{array}\right.
$$

then $A=\left(a_{n k}\right) \in\left(c_{0}, c\right)$ since $\lim _{n} \sum_{k=1}^{\infty} a_{n k} y_{k}=\lim _{n} \sum_{k=1}^{n} a_{n k} y_{k}$ exists for all $y \in c_{0}$ (23). This implies that 
$\operatorname{Sup}_{n} \sum_{k=1}^{\infty}\left|a_{n k}\right|=\operatorname{Sup}_{n} \sum_{k=1}^{n}\left|\sum_{i=k}^{n} a_{i}\right|<\infty$ [4]. Thus we get $\sum_{k=1}^{\infty}\left|R_{k}\right|<\infty$. Furthermore (2.2) implies that limn $R_{n+1} x_{n+1}$ exists for each $x \in c_{0}(\Delta)$ then we get $\left(R_{n}\right) \in c_{0}^{N}(\Delta)$ by lemma 1. This completes the prool.

THEOREM 1. $A=\left(a_{n k}\right) \in\left(c_{0}(\Delta), c\right)$ iff

$T_{1} \cdot\left(R_{n k}\right) \in c_{0}^{N}(\Delta)$, for each $n \in \mathbb{N}$

$T_{2} \cdot R=\left(R_{n k}\right) \in\left(c_{0}, c\right)$

Proof. If $a \in\left(c_{0}(\Delta), c\right)$ then the series $A_{n}(x)=\sum_{k=1}^{\infty} a_{n k} x_{k}$ are convergent for each $n \in \mathbb{N}$ and for all $x \in c_{0}(\Delta)$, this implies that $\operatorname{Sup}_{n} \sum_{k=1}^{\infty} l a_{n k} \mid<\infty$ and $\lim _{n} \sum_{k=p}^{\infty} a_{n k}=a_{p}$ exists for each $p \in \mathbb{N}$ [3] (page 166). From lemma 3 we have $\sum_{k=1}^{\infty}\left|R_{n k}\right|<\infty, \lim _{k} R_{n k} x_{k}=0$ for each $n \in \mathbb{N}$ and for all $x \in\left(c_{0}(\Delta)\right.$. This proves $T_{1}$. If we write again (2.2) we get

$$
\sum_{k=1}^{m} a_{n k} x_{k}=\sum_{k=1}^{m+1} R_{n k}\left(x_{k}-x_{k-1}\right)-R_{n m+1} x_{m+1}
$$

and

$$
A_{n}(x)=\sum_{k=1}^{\infty} a_{n k} x_{k}=\sum_{k=1}^{\infty} R_{n k}\left(x_{k} \cdot x_{k-1}\right)
$$

This shows that $R \in\left(c_{0}, c\right)$. If we use again lemma 3 and (2.5) we get the sufficiency of $T_{1}$ and $T_{2}$. Similarly we can prove that

i) $A \in\left(c_{0}(\Delta), c_{0}\right)$ iff $T_{1}$ and $R \in\left(c_{0}, c_{0}\right)$

ii) $A \in\left(c_{0}(\Delta), I_{\infty}\right)$ iff $T_{1}$ and $R \in\left(I_{\infty}, I_{\infty}\right)$

iii) $A \in\left(c_{0}(\Delta), M_{0}\right)$ iff $T_{1}, R \in\left(I_{\infty}, I_{\infty}\right)$ and

$$
B=\left(b_{n k}\right)=\left(a_{n k}-a_{n k+1}\right) \in\left(c_{0}(\Delta), c_{0}\right)
$$

iv) $A \in\left(c_{0}(\Delta), c_{0}(\Delta)\right)$ iff $\left(a_{n k}\right) \in c_{0}^{\beta}(\Delta)$, for each $n \in I N$ and $C=\left(c_{n k}\right)=\left(a_{n k}-a_{n-1, k}\right) \in\left(c_{0}(\Delta), c_{0}\right)\left(a_{o k}=0\right)$

Open questions

1) Matrix maps for $M_{0}$.

2) $M_{0}$ has a Schauder basis with $\left\|_{\Delta}\right\|_{\Delta}$ It is $\left(E_{k}\right)$. (we can write $x=\sum_{k=1}^{\infty}\left(x_{k}-x_{k-1}\right) E_{k}$, $\theta$ ach $x \in M_{0}$ ) Then $\left(M_{0}, \|_{\Delta}\right)$ is separable.

Is $M_{0}$ separable or have a Schauder basis with $\|.\|_{\infty}$ ?

3) It is obvious that $c_{0} \subset c \subset M_{0} \subset I_{\infty}$ and inclusions are strict. In this order, is there a separable space $E$ which is $\propto E C I_{\infty}$ with the norm $\|.\|_{\infty}$ ? If not, is $c$ an upper bound according to separability?

\section{REFERENCES}

[1] Garling D.J.H., The $\alpha$-and $\beta$-duality of sequence spaces, Proc. Comb. Phil soc. 1967 63(963-981)

[2] Kızmaz H., On Certain Sequence Spaces, Canad. Math. Bull. 25(2) 1981 (169-176)

[3] Maddox I.J., Elements of Functional Analysis Cambridge 1970

[4] Stieglitz M. - Tietz H., Matrixtransformationen Von Fongenraumen Eine Ergebnisübersicht Math. Z. 154 (1977) $(1-16)$ 


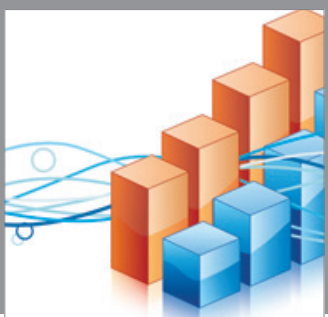

Advances in

Operations Research

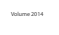

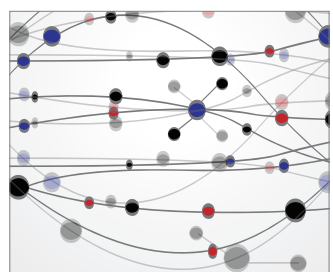

\section{The Scientific} World Journal
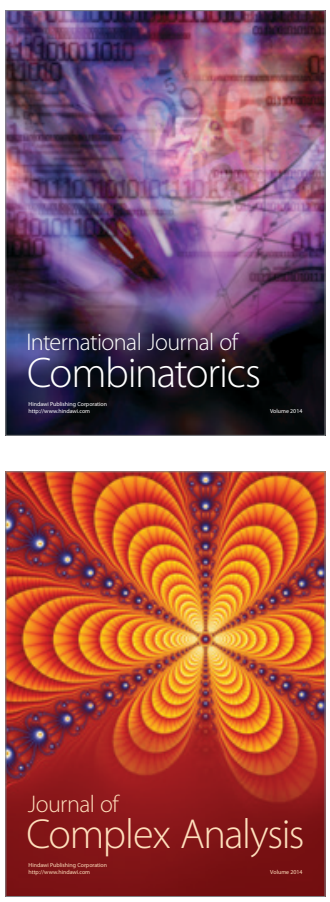

International Journal of

Mathematics and

Mathematical

Sciences
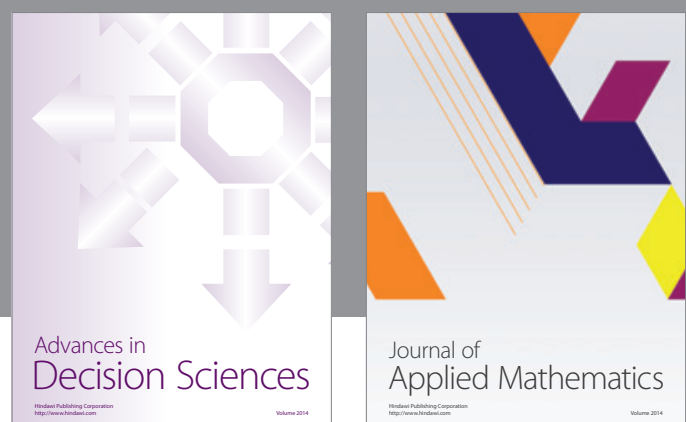

Journal of

Applied Mathematics
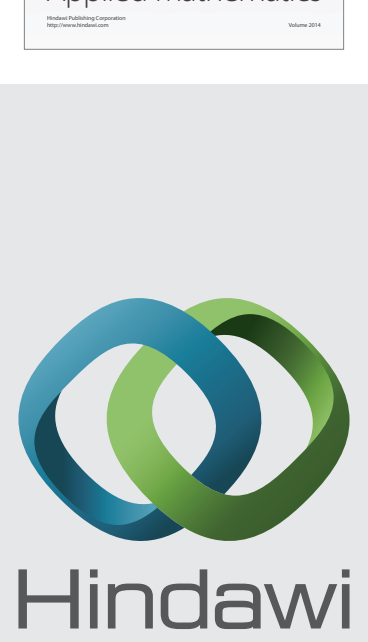

Submit your manuscripts at http://www.hindawi.com
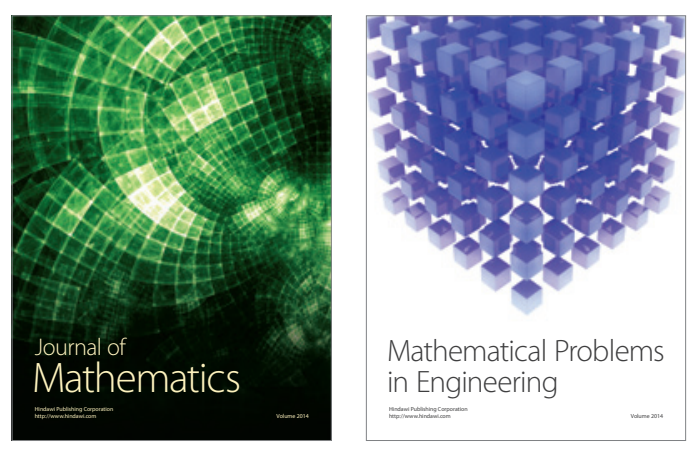

Mathematical Problems in Engineering
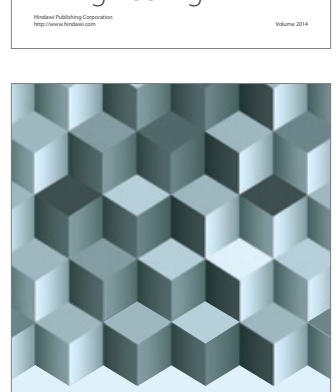

Journal of

Function Spaces
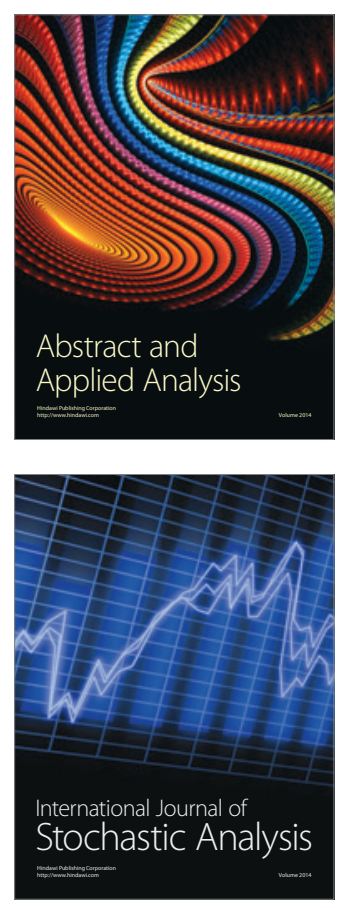

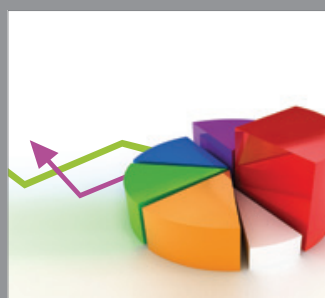

ournal of

Probability and Statistics

Promensencen
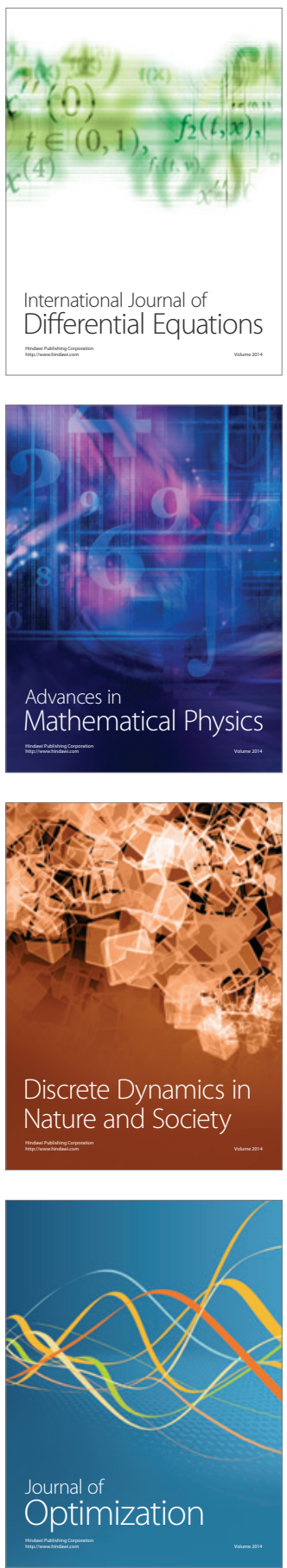